\title{
Design of Lower Limbs Rehabilitative Robot
}

\author{
Xinqi $\mathrm{Lu}^{1, \mathrm{a}}$ Mianhao Zhang ${ }^{2, \mathrm{~b}}$ Liulin $\mathrm{Wu}^{3, \mathrm{c}}$ \\ ${ }^{123}$ College of Engineering of Zhejiang Normal University, School of Vocational Technical Education, \\ Zhejiang, Jinhua 321004 \\ A452602516@qq.com, ${ }^{\mathrm{b}} 670570089 @ q q . c o m,{ }^{c} w u l i u l i n 0810 @ 163 . c o m$
}

Keywords: lower limbs rehabilitative robot; adjustable; compact

Abstract. This paper designs a lower limbs rehabilitative robot with compact, functional and widely applied features for patients with lower limbs disorder. This robot is consisting of hip transverse adjustment mechanism, hip and knee joint compound movement mechanism, bed angle adjustment mechanism and pedals. This robot is small and convenient to store and remove, which is suitable for replace in the bedroom of patient. This robot can simulate the walking and running activities, and adjust the size according to the patient's height and build, expanding the scope of application. Rehabilitation training is a long term process, and this robot can reduce the working load of physical therapist and resource cost, and improve the rehabilitation training effectiveness and effect compared with traditional rehabilitation training method.

\section{Introduction}

In recent years, with the degree of internal growing aging population, the patients with lower extremity nerve injuries due to frequent traffic accidents and sports accidents are increased gradually. The injuries of lower limbs can lead to hemiplegia and paralysis which will influence the physical and mental health of the patient as well as impose the pressure of family and society. Therefore, how to recover the moveable function of patients uttermost is the urgent and important content in clinical rehabilitation.

It is clinically proven that the patients with lower limbs movement disorder can improve the rehabilitation effect of movable function through scientific and reasonable rehabilitative training, except adopting traditional drug therapy and operation.

However, due to the limited resource of therapist and high medical expenses, some patients missed the best time of treatment. Otherwise, in the process of human treatment lacking the strict inspection and control, therefore, the rehabilitation training simply relying on the therapist cannot satisfy the amount of patient in the society. The rehabilitative robot raised in recent years can help to make the rehabilitation training of lower limbs for patients, especially robot-assisted walking training are gradually become the key point of research both in home and abroad.

The lower limbs rehabilitative robot in this paper can imitate the test of system to give feedbacks and make effective medical plan with great rehabilitation effect. It is compact, adjustable and suitable for widely population. It can reduce the need of physical therapist resources and cost, as well as improve the rehabilitation effectiveness.

\section{Main structure design of lower limbs rehabilitative robot}

The structure design of lower limbs rehabilitative robot is adopted the hip adjustment mechanism shown in Figure 1, hip and knee joint compounding movement mechanism shown in Figure 2 to adjust the position of each knee during the process of training and finish the rehabilitation training of limbs, and the mechanisms shown in Figure 3 and 4 to adjust the angle and height of bed in order to realize the rehabilitation training of patients with lower limbs disability and in a comfortable position. 


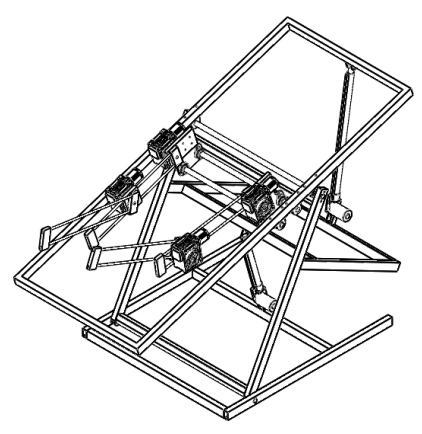

1. Hip transverse adjustable mechanism

There is a location hole above the convex connecting plate 1 shown in Figure 1 used for connecting the compound movement mechanism 4 of knees and hip; beside the convex connecting plate, there are two table supports 2 , and below the convex connecting plate, there are two holes cooperating with movable straight-line rail 3, achieving the function of hip transverse adjustable mechanism.

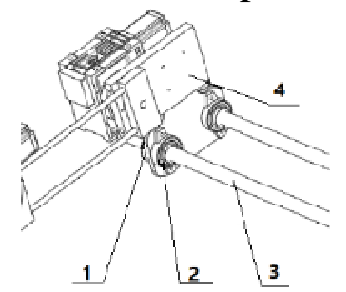

Figure 1 Hip transverse adjustable mechanism

1-convex connecting plate, 2table supports, 3-movable straight-line rail,

4-ompound movement mechanism

2.Hip and knees joint compounding movement mechanism

The hip and knees joint compounding movement mechanism shown in Figure 2 are used for completing the activity of limbs joints, mainly connecting the fixed connecting plate 4 with convex connecting plate 1 in Figure 1; there is a hole in fixed connecting plate 4 used for cooperating with speed reducer 2 with 57 motor 1, speed reducer 2 has an output shaft matching with the holes above guide rail 5 to transfer the torque, one side of hip guide rail sticks to fixed connecting plate 6; there is a movable slider 6 on hip guide rail 5 to fixed the position through set screws, the installation of slider 6 is similar with above mention content, fix the speed reducer 22 with 57 motor 12 through slider 3 , then transfer the torque to movable rail 5 through output shaft, the hip and knees joint rehabilitation activities can be completed through the control of angle with different time.

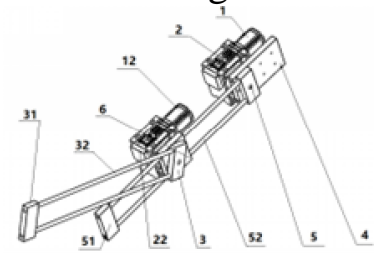

Figure 2 Hip and knees joint compounding movement mechanism

1-convex connecting plate, 2-speed reducer, 3-slider, 4- fixed connecting plate ,

5-hip guide rail, 6-hip guide rail, 12- motor , 22-speed reducer

\section{Angle Adjustment Mechanism of Rehabilitation Equipment Body}

The angle adjustment mechanism of lower limb rehabilitation equipment as shown in Figure 3 and Figure 4 is used to adjust the angle of equipment body to allow the patient to lay flat and make lower limb rehabilitation exercise. A rotation axis passes the through-hole which is in the front side of chassis frame 1 in Figure 3 and fixes the axis through the middle frame bottom 33 to form a revolute joint, and the top of middle holder 34 and the middle part of equipment body 5 form a revolute joint. 


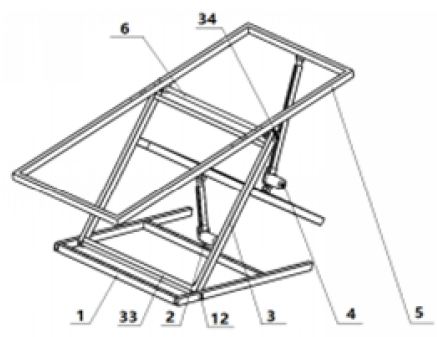

Figure 3 Angle Adjustment Mechanism of Rehabilitation Equipment Body（a ） 1-chassis frame , 2-linear actuatorr, 4-linear actuator, 5-equipment body, 12-Rotation axis, 33-middle frame bottom, 34- top of middle holder

The support frame of the middle frame 31 in Figure 4 fixes the linear actuator 2 and vice versa. When the linear actuator 2 is moving, the angle of whole equipment body changes slightly; the support frame of the middle frame 32 is connected with the middle part of equipment body 5 through the linear actuator 4 , and when the linear actuator 4 is moving, the angle of the equipment body will have a great change for completing the angle adjustment of the equipment body and allowing the patient to lay flat to make low limb rehabilitation exercise.

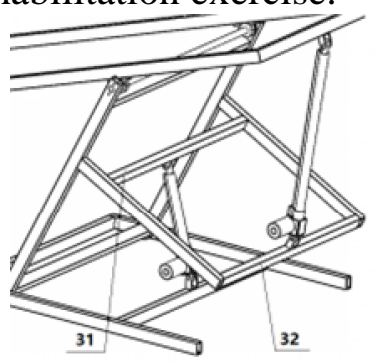

Figure 4 Angle Adjustment Mechanism of Rehabilitation Equipment Body（b ) 31-middle frame , 32-middle frame

\section{Foot Pedal Mechanism of Lower Limb Rehabilitation}

In the foot pedal mechanism of lower limb rehabilitation as shown in Figure 5, the foot pedal 2 is connected with guide shaft 1 and can slide up and down along the guide shaft 1 to be suitable to users with different heights.

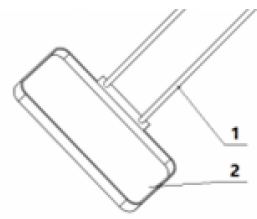

Figure 5 Foot Pedal Mechanism of Lower Limb Rehabilitation 1- guide shaft, 2-foot pedal

\section{Design of Process Control}

Arduino MEGA 2560 is applied in the design to realize a control with multi-joint axis. The basic control diagram is shown in Figure 6. The input part of Arduino includes the control to the equipment body height, equipment body angle, joint movement speed and range of joint motion. After the input signal is sent to Arduino, Arduino will control the joint movement speed, range of joint motion, rise and fall and flipping of equipment body. 


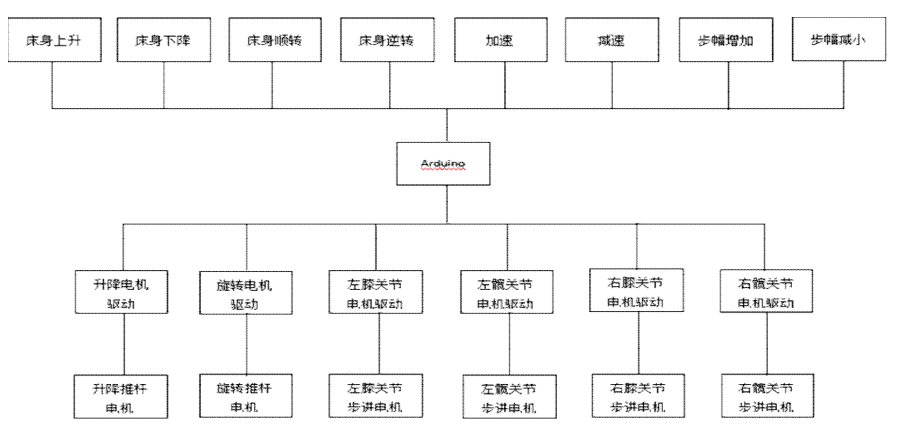

Figure 6 Control diagram

\section{Conclusion}

With a lower limb rehabilitation robot as an example in this article, a rehabilitation robot applicable to the lower limb rehabilitation is developed for patients whose lower limbs are difficult to move and who want to get recovered quickly. Because the traditional lower limb rehabilitation equipment is only suitable to people in good state of lower limb recovery and has single function, this article improves the traditional lower limb rehabilitation equipment and designs an overall structure of rehabilitation robot, mainly including the structural design of joint, equipment body and holder; it also uses the three-dimensional modeling to realize the mechanism optimization, introduces the Arduino process diagram and applies Arduino program to control the joint motors and lifting motors and to realize the design of rehabilitation robot at the end. This designed mechanism is simple and has a compact structure, and its adjustable structure is widely applicable to people. It reduces the required therapists resource and cost and improves the efficiency of rehabilitation.

\section{References}

[1] Research on Movement Control Method of Omni-directional Lower Limbs Rehabilitation Robot [D]. Jiang Ying. Shenyang University of Technology 2013

[2] Rehabilitation Robot Prototype Development and Gait Control Research [D]. Wang Lingjun. Harbin Institute of Technology 2010

[3] Research on Wearable Lower Limbs Rehabilitation System [D]. Shi Lei. Xihua University 2012

[4] Mechanism Analysis and Optimal Design of Wearable Lower Limbs Rehabilitation Robot [D]. Deng Chuhui. Beijing University of Technology 2012

[5] Sun Baoheng. Lower Limbs Rehabilitation Robot Based on Bionic Joint [P]. Chinese Patent No.: CN104688492A,2015-06-10.

[6] $\mathrm{Xu}$ Zhenhua. Knee Joint Device of Rehabilitation Robot [P]. Chinese Patent No.: CN205612703U,2016-10-05.

[7] Wang Yong; Gao Aili. Laying and Standing Rehabilitation Robot with Multiple Adjustable Angles [P]. Chinese Patent No.: CN105496726A,2016-04-20.

[8] Ji Aihong; Wang Wei. Parallel Linkage Lower-limb Rehabilitation Robot and Its Working Methods [P]. Chinese Patent No.: CN105963102A,2016-09-28.

[9] Chen Diansheng; Ning Meng; Zhang Benguang. Lower-limb Exoskeleton Rehabilitation Robot for Working Assistance [P].ChinesePatent:CN104490568A,2015-04-08.

[10] Wei Jiangjun; Xiong Tao; Zhang Ruixing; Liu Zuokai; Guo Hua. Lower Limbs Rehabilitation [P]. Chinese Patent No.: CN205411569U,2016-08-03. 